Cuadernos de Investigación Geográfica. Tomo IX-1983 - págs. 83-98

(C) Colegio Universitario de La Rioja - Logroño

\title{
LOS VIÑEDOS RIOJANOS DURANTE LA SEGUNDA MITAD DEL SIGLO XIX
}

\author{
Pedro García Santamaría
}

\begin{abstract}
RESUMEN
Se estudia la evolución del viñedo riojano durante la segunda mitad del siglo XIX y se explican las razones que justifican la rápida expansión superficial, entre las que cabe citar las enfermedades de la vid, el trazado del ferrocarril y las exportaciones de vino a Francia durante la crisis de la filoxera.
\end{abstract}

\section{SUMMARY}

The evolution of the Riojan vineya*d during the second half of the 19th century is studied, and the reasons that justify the rapid superficial expansion are explained; among them, we can mention the diseases of the vine, the running of the railway and the exports of wine to France during the phylloxera crisis.

Se pretende en las páginas siguientes resaltar el conjunto de factores que se desencadenaron de forma sincrónica en el último tercio del siglo XIX, haciendo posible la aparición, por vez primera, del vino de Rioja con los caracteres de calidad y envejecimiento que hoy conocemos. Del conjunto de los factores destacaremos las enfermedades de la vid y en especial la filoxera, que además de revalorizar nuestros viñedos por el temprano arrasamiento de los viñedos franceses, hizo posible que en La Rioja se llegaran a conocer los métodos bordeleses para la elaboración del vino.

El primer problema con que nos enfrentamos es el de la carencia y precariedad de las fuentes de información; el rigor científico y la exactitud del Catastro del Marqués de la Ensenada no se va a superar en España hasta muy entrado el siglo XIX. Las publicaciones del siglo XIX especializadas en temas agrarios se alarman ante la falta de previsión y desconocimiento estadístico 
que ofrecen los organismos competentes en todo lo referente al cultivo, recolección y comercio de la vid. Buena prueba de dicha situación son las siguientes líneas extraidas de la revista agrícola Los vinos y aceites (QuincenalBiblioteca Nacional. 5. 5.634):

"En cualquier país donde el servicio de estadística agrícola esté bien organizado, se poseen en la época en que nos encontramos del año, datos más que suficientes para poder juzgar cualquier industria. En Francia, en Austria, en Italia, en Portugal, en una palabra, en todas las naciones vitícolas, saben ya de seguro cuál ha sido la producción de vino de las diferentes clases y en las diferentes comarcas. En España no sabemos tal cosa aún, ni la sabremos nunca, si en la gestión oficial confiáramos; pero, por fortuna, hay en nuestro país más iniciativa individual de la que especialmente se cree... pues a esta iniciativa se debe el que podamos conocer con bastante aproximación la cosecha del presente año $1879 . . . "$

Ante esta situación, siempre que se aporten datos de extensión del viñedo a escala provincial, deberán ponerse en entredicho, ya que en muy pocas ocasiones los archivos de los diferentes organismos oficiales logran ponerse de acuerdo. A pesar de este grave inconveniente y partiendo de los archivos locales y revistas especializadas en temas económicos-agrarios de la época, aportaremos unos datos que resultan más fiables que las estadísticas oficiales.

\section{EI viñedo tradicional}

\section{Evolución y distribución}

El viñedo ha formado parte tradicionalmente del paisaje agrario de La Rioja. Sin adentrarnos en pormenores históricos, que no son objeto específico de estas líneas, convendría resaltar el empuje del viñedo durante toda la alta Edad Media, al unísono con la carencia de circuitos comerciales en los territorios cristianos peninsulares y con el papel jugado por el vino en la subsistente economía del alto medievo, sin descartar su ineludible vinculación con las celebraciones eucarísticas y el gran afán demostrado por este cultivo en los poblados monasterios de la época.

A partir de los siglos XII y XIII el viñedo retrocede, abandonando las tierras más altas y asentándose progresivamente en el somontano y núcleos ribereños. La reactivación comercial de la Baja Edad Media, en especial los mercados comarcales que ponen en contacto la montaña con la llanura, permite el que una buena parte de los municipios riojanos montañosos puedan adquirir el vino en las cabeceras de las comarcas sin tener que llegar a cultivarlo de forma residual para tratar de autoabastecerse.

Estas pautas vitícolas bajo-medievales, al amparo de una fácil colocación del viñedo en los núcleos ibéricos sorianos y burgaleses, se consolidan a lo 


\section{LOS VIÑEDOS RIOJANOS}

largo del siglo XVI, dando como resultado que un término como Calahorra ${ }^{1}$ fuera durante el último tercio del siglo XVI el primer productor de vino de La Rioja.

A mediados del siglo XVIII el conjunto del paisaje agrario riojano se va configurando casi definitivamente, por lo que a cultivos tradicionales respecta. Los núcleos ribereños, como Calahorra ${ }^{2}$, se van especializando en cultivos cerealistas y se dibujan ya las áreas de claro predominio vitícola. En el año 1750 los diez municipios que contaban con mayor superficie de vid eran los siguientes ${ }^{3}$ :

$\begin{array}{llcc}\quad \text { Población } & \text { Regadío } & \text { Secano } & \text { Total fanegas } \\ 1^{\circ} \text { Alfaro } & 5463 & - & 5463 \\ 2^{\circ} \text { S. Vicente } & - & 5349 & 5349\end{array}$

1. Producciones medias de algunas villas de La Rioja Baja. Extraidas del A.H.N. de Simancas (Expedientes de Hacienda siglo XVI. Sección XV. Cifras medias para el periodo 1579-1585).

\begin{tabular}{|l|c|c|c|c|c|c|c|c|}
\hline & Población & $\begin{array}{c}\text { Vino } \\
\text { (cántaras) }\end{array}$ & $\begin{array}{c}\text { Trigo } \\
\text { (Fanegas) }\end{array}$ & $\begin{array}{c}\text { Cebada } \\
\text { (Fanegas) }\end{array}$ & $\begin{array}{c}\text { Centeno } \\
\text { (Fanegas) }\end{array}$ & $\begin{array}{c}\text { Avena } \\
\text { (Fanegas) }\end{array}$ & $\begin{array}{c}\text { Olivas } \\
\text { (Fanegas) }\end{array}$ & Corderos \\
\hline Agoncillo & & 2155 & 3000 & 2000 & 100 & 200 & & 504 \\
Alcanadre & 194 & 4453 & 2500 & 2400 & 730 & 130 & 170 & \\
Arnedillo & 180 & 4341 & 1200 & 100 & 120 & 150 & 80 & 800 \\
Arrubal & & 700 & 1000 & 800 & 10 & 150 & & 455 \\
Ausejo & & 19455 & 10800 & 6875 & 500 & 480 & 200 & 2400 \\
Autol & 364 & 8785 & 7470 & 3960 & 1800 & & 110 & 2560 \\
Calahorra & 1074 & 73050 & 33300 & 16620 & 7420 & 1350 & 3000 & 6200 \\
Clavijo & & 7696 & 3400 & 1600 & 580 & 850 & & \\
Herce & 363 & 5520 & 1500 & 350 & 400 & & 450 & 1000 \\
Jubera & & 3361 & 6800 & 2700 & 2500 & 500 & 600 & 1200 \\
Lagunilla & & 3410 & 3375 & 2675 & 1290 & 120 & 150 & 700 \\
Murillo de R.L. & & 24300 & 3500 & 4000 & 600 & 1000 & 50 & 2630 \\
\hline
\end{tabular}

2. Archivo Histórico Provincial de Logroño. Catastro del Marqués de la Ensenada. Tomos 166 a 188. Distribución de los principales cultivos de Calahorra en el año 1751:

\begin{tabular}{|l|c|c|}
\hline Conceptos & Regadio (fanegas) & Secano (fanegas) \\
\hline Hortaliza & 489 & \\
Cereal & 12535 & 6440 \\
Vid & 1700 & 5 \\
Olivar & 599 & 5 \\
\hline
\end{tabular}

3. Datos obtenidos a partir de un estudio individualizado del Catastro del Marqués de la Ensenada. 
PEDRO GARCIA SANTAMARIA

$\begin{array}{lllll}3^{\mathrm{a}} \text { Haro } & - & 5289 & 5289 \\ 4^{\mathrm{a}} & \text { Briones } & - & 5066 & 5066 \\ 5^{\mathrm{a}} \text { Arnedo } & - & 4200 & 4200 \\ 6^{\mathrm{a}} \text { Logroño } & 2342 & 1448 & 3790 \\ 7^{\mathrm{a}} & \text { Fuenmayor } & 285 & 3078 & 3363 \\ 8^{\mathrm{a}} \text { Autol } & 20 & 3334 & 3354 \\ 9^{\mathrm{a}} & \text { S. Asensio } & - & 3100 & 3100 \\ 10^{\mathrm{a}} \text { Cenicero } & - & 2681 & 2681\end{array}$

Los últimos años del siglo XVIII y primeros del XIX continúan con una tónica similar, si bien la agricultura va marginando la actividad ganadera en una buena parte de La Rioja ${ }^{4}$. En la comarca de Haro existió auténtica fiebre vitícola; en el año 1792 la autoridad municipal intentó recuperar los comunes que habían sido puestos en cultivo abusivamente; sin embargo, a instancias superiores se admitieron las plantaciones furtivas, prohibiendo el arranque masivo de las mismas.

El conjunto de los viñedos riojanos evoluciona de forma dispar hasta la segunda mitad del siglo XIX; mientras que el sector noroccidental de la región se consolida como el núcleo vitícola de mayor entidad de La Rioja, La Rioja Central y Baja alcanzan una mayor especialización cerealista. A continuación se incluye la extensión de los viñedos y del cereal en un conjunto de municipios riojanos en el año 1751, confirmándose así el predominio alcanzado por el cereal en una buena parte de La Rioja:

\begin{tabular}{|l|c|c|}
\hline Municipio & Cereal (fanegas) & Vid (fanegas) \\
\hline Albelda & 2337 & 129 \\
Sorzano & 2270 & 20 \\
Baños de Rioja & 1850 & 20 \\
Azofra & 2500 & 500 \\
Canillas & 1200 & 32 \\
Murillo de R.L. & 7332 & 2059 \\
Arrubal & 1100 & 26 \\
Cervera & 4070 & 682 \\
Bergasa & 1000 & 200 \\
Alfaro & 15750 & 5463 \\
\hline
\end{tabular}

4. Archivo Histórico Nacional. Leg. 31.231. n ${ }^{\circ}$. Folio 40. Año 1784. 


\section{LOS VIÑEDOS RIOJANOS}

Dos hechos marcan el proceso evolutivo del viñedo riojano a lo largo del siglo XIX; el incremento del viñedo y el generalizado abandono del mismo en las areas de regadío, donde tienden a introducirse más los cereales y los cultivos de huerta. Si a mediados del siglo XVIII los viñedos de regadío alcanzaban 4.000 Has. de las aproximadamente 17.000 cultivadas, en 1875 existían en La Rioja 24.384 Has. de viñedo, y de ellas sólo 2.441 eran de regadío y 21.932 de secano ${ }^{5}$. Es decir,entre ambas fechas el viñedo de regadío había pasado de representar el $23,5 \%$ al $10 \%$ de la superficie total ocupada por la vid.

En el año 1878 varias fuentes consultadas otorgan una producción global de $312.318 \mathrm{Hl} ., 12 \mathrm{HI}$. por hectárea, que en principio no superan ni con mucho la rentabilidad que en 1751 otorgaba el Catastro del Marqués de la Ensenada. A pesar de las tergiversaciones del Catastro, voluntarias o no, los viñedos regables eran más abundantes en el siglo XVIII y las plantaciones se seguían llevando a cabo bajo métodos tradicionales. En el año 1877, el rendimiento medio por hectárea era en España de 17,30 Hl., cuando en La Rioja apenas se superan los 12; los cinco hectolitros de diferencia, además de los propios errores estadísticos y de documentación, habrá que buscarlos en la escasa transformación sufrida por el agro riojano.

En los últimos años del siglo XIX la evolución superficial es la siguiente. En el año 1881 se otorgan a La Rioja 55.174 Has. de viñedo; dado que es precisamente entre 1880 y 1890 cuando se produce el momento álgido de nuevas plantaciones, creemos que la cifra desborda a la realidad. En el año 1885 existen dos fuentes informativas que suministran datos sobre la extensión del viñedo en la provincia; el Ministerio de Agricultura otorgaba en ese año 34.684 Has. de viñedo, mientras que las revistas especializadas cifraban su valor en $31.608^{6}$. Aun cuando esta segunda nos parece baja, ambas son más fiables y resultan más lógicas que la apuntada para 1881.

En el año 1889 el viñedo riojano alcanza 52.390 Has., de las cuales 4770 eran de regadío y 47690 de secano ${ }^{7}$. El viñedo de regadío —que en 1874 era de 2.448 hectáreas y que había sufrido un gran retroceso con respecto a las cifras que de él se tenían en el siglo XVIII- se incrementa de nuevo hasta superar los viñedos regables de 1750. La respuesta a este incremento es fácil de encontrar; si se analizan las cifras de exportación de vino a Francia, se tiene en consideración la proximidad de Irún y la trayectoria recorrida por el ferrocarril, que atraviesa los puntos vitales de la producción vitícola riojana, amén

5. Mernoria sobre el estado de la agricultura e industria derivada Provincia de Logroño. Consejo superior de Industria y Comercio. 1876.

6. La crisis agrícola y pecuaria. Madrid 1887-1889. Siete volúmenes. Biblioteca Nacional. 1. 16769 y siguientes.

7. Avance estadístico sobre cultivos y producción de la vid en España, formado por la Junta Consultiva Agronómica. 1889. Madrid, 1891. 195 páginas. 
de la facilidad de venta y cobro del vino por el asentamiento de comerciantes franceses en muchos de nuestros pueblos, se encontrará la explicación del nuevo impulso cobrado por las plantaciones de viñas en tierras de regadío.

En el mismo año, 1889, y de acuerdo con la misma fuente, el viñedo riojano se distribuía entre sus partidos judiciales de la manera siguiente:

\begin{tabular}{|l|c|c|}
\hline Partido & Secano (Ha.) & Regadío (Ha.) \\
\hline Alfaro & 2690 & 500 \\
Arnedo & 3600 & 800 \\
Calahorra & 2800 & 420 \\
Cervera & 1200 & 500 \\
Haro & 16530 & 800 \\
Logroño & 10000 & 500 \\
Nájera & 9200 & 602 \\
Sto. Domingo & 1600 & 650 \\
\hline
\end{tabular}

Las cepas predominantes en La Rioja Alta, según la misma fuente de información, diferían bastante de La Rioja Baja; graciano, garnacho, mazuelo y colgadera, eran las fundamentales en La Rioja Baja, mientras que el tempranillo era casi exclusivo en la Alta, acompañado en ocasiones del graciano y del mazuelo.

En 1890, el archivo del Ministerio de Agricultura concede a los viñedos riojanos una extensión de 47.683 Has. De nuevo las revistas agrícolas están en desacuerdo con la citada cifra; El avance estadístico sobre cultivos de la vid en España otorga a nuestros viñedos 52.390 Has.

En toda La Rioja había nacido una honda preocupación por incrementar la producción y el comercio del vino, de tal forma que al Congreso de 1886, celebrado en Madrid, asistieron un buen número de localidades productoras de vino: Logroño, Haro, Nájera, Cervera, Calahorrá, Alfaro, Arnedo, Alberite, Cenicero, Entrena, Fuenmayor, Lardero, Murillo de Río Leza, Navarrete y Sorzano, se encontraban entre ellas. Por la importancia del cultivo para la provincia también acudieron las personalidades y organismos riojanos vinculados con el vino, Juan Domingo Santa Cruz, Sagasta, Tirso Rodríguez, Eduardo Peralta, Amós Salvador, Protásimo Gómez, Marqués de Riscal, Marqués de Murrieta, Galo Pobes y la Junta de Labradores de Logroño que era representada por el Marqués de Murrieta.

La producción de algunas localidades en los últimos años del siglo pueden resultar interesantes, no sólo por la inmediata llegada de la filoxera, sino por- 


\section{LOS VIÑEDOS RIOJANOS}

que todo estudio vitícola posterior a 1904 deberá tener en cuenta la extensión y producción de los viñedos a finales del siglo $\mathrm{XIX}^{8}$ :

\begin{tabular}{|l|c|c|}
\hline Municipios & 1897 & 1898 \\
\hline Haro & 110.000 cántaras & 137.000 cántaras \\
Leiva & $24.000 \quad$, & $44.000 \quad "$, \\
Arnedo & & $123.000 \quad$, \\
Ollauri & $60.000 \quad$, & \\
Abalos & & $61.644 \quad "$ \\
Uruñuela & $57.000 \quad$, & \\
\hline
\end{tabular}

\section{La calidad del vino riojano hasta fines del siglo XIX}

Es conveniente recordar la escasa entidad de los vinos cosechados en nuestra Comunidad durante casi todo el siglo XIX; ni una sola de las publicaciones especializadas en temas agrarios que pueblan las estanterías de los archivos nacionales hacen especial mención del vino riojano durante el siglo XIX.

Todo el conjunto de adjetivos glorificantes y leyendas seudomitológicas, a las que tan a menudo nos han tenido acostumbrados algunas plumas deben dejar paso a la más cruda realidad, ya que todas las celebraciones votivas importantes iban siempre acompañadas, en Logroño, de la consiguiente adquisición de vinos blancos procedentes del Valle del Duero. Si a la ignorancia comercial de nuestros vinos unimos su carencia de personalidad, amén de las limitaciones técnicas de los vitivinicultores, que ni tenían medios ni eran capaces de conservar el vino más de una añada, tendremos una visión aproximada de la vitivicultura riojana a mediados del siglo XVIII.

La máxima extensión del viñedo decimonónico coincide cronológicamente con la aparición del Vino de Rioja, a finales del siglo XIX. Partiendo de los oscuros vinos autóctonos, se asimilarán rápidamente por algunos bodegueros los métodos bordeleses de envejecimiento y conservación; estos métodos fueron expuestos por los vinateros franceses establecidos en La Rioja para adquirir vino durante el tiempo en que sus campos fueron arrasados por la filoxera. Sin embargo, el proceso de aparición del "Rioja" es mucho más complejo y en él intervienen factores tan importantes como el aprendizaje al que se sometieron técnicos riojanos en Burdeos, la creación de la Estación Enoló-

8. Revista vinícola de Agricultura. Publicada en Zaragoza a partir del año 1871. Biblioteca Nacional. 6. 7126. 


\section{PEDRO GARCIA SANTAMARIA}

gica, que fue resolviendo los múltiples problemas técnicos planteados, la inversión de capital foráneo en las bodegas prefiloxéricas, etc.

\section{Los factores desencadenantes del cambio}

\section{Las enfermedades de la vid}

Las enfermedades de la vid constituyen el problema básico de la agricultura riojana en la segunda mitad del siglo XIX: este hecho es el que en última instancia revolucionará el sector vitivinícola y potenciará el conjunto de la agricultura riojana, primero a escala internacional, por el arrasamiento de los viñedos franceses, y más tarde dentro de España, por ser La Rioja uno de los últimos enclaves en ser invadido por la filoxera. El impacto de esta última será analizado en un apartado especial.

El oídio de la vid, la primera de las enfermedades, es de origen americano. Procedente de Estados Unidos llega a Orense, a través de Portugal, en 1850 , provocando en un corto periodo de tiempo la ruina de las vides en las regiones húmedas. En seguida se conocerá el tratamiento del azufre, recuperándose los viñedos afectados y comenzando a partir de 1865 uno de los períodos más florecientes de los viñedos hispanos.

En La Rioja, el oídio afecta a las viñas de forma brusca entre 1855 y 1862. Para tener una idea de lo que supuso esta enfermedad en algunos lugares de la región hemos tomado el ejemplo de Calahorra, donde se pasó de una producción de 62.590 cántaras en el año 1852 a 48.711 en 1854 y a 24.140 en 1859; Haro, 200.000 cántaras en 1850 y 43.000 en 1858. En Logroño la cosecha del año 1862 fue prácticamente nula.

El temor a ver devastados los viñedos supuso un gran avance en la utilización de las cepas resistentes al oídio; en La Rioja numerosos viticultores plantaron la variedad garnacha, aun cuando no da un fruto tan fino como el tradicional, e incluso el tempranillo. Especialmente los sarmientos garnachos se exportaron a diversas provincias españolas, sobre todo a aquellos lugares donde la viticultura estaba relacionada con el vino blanco.

A partir de estos años y hasta finales de siglo, los viñedos riojanos conocen una época de prosperidad, sólo empañada por la expansión del mildeu, que repercutiría negativamente en las cosechas de los años 1886 y 1887 . El mildeu, que aparece en 1885 en Galicia y en el Valle del Ebro, repercute ese mismo año en la cosecha de La Rioja Alta. En 1885, la mitad de las viñas de Logroño no pudieron ser cultivadas normalmente. Los salarios descendieron y los precios subieron abusivamente. 


\section{LOS VIÑEDOS RIOJANOS}

\section{La vía férrea}

El incremento del viñedo y el auge comercial no habrían podido consumarse con el sistema de transporte tradicional del vino; los arrieros, con sus limitaciones técnicas y temporales, condujeron el vino a lo largo de los siglos hasta los núcleos ibéricos burgaleses y sorianos, a las provincias vascongadas e incluso, en ocasiones hasta Cantabria. A partir de 1860 se pone en marcha la línea Bilbao-Barcelona, que sería fundamental para la salida de nuestros vinos; esta vía férrea se adentra en La Rioja por las Conchas de Haro y hasta Alfaro recorre los puntos de mayor especialización vitícola. Las exportaciones del vino no pueden entenderse separadas del ferrocarril; después de 1870 , el ferrocarril se vincula de tal forma con viticultura que los precios del vino de cada uno de los municipios van a sufrir considerables variaciones según sea su situación con respecto a la estación más cercana.

\section{Las exportaciones a Francia}

A medida que los viñedos franceses son arrasados por la filoxera, los comerciantes franceses vienen a instalarse a La Rioja en busca de unos vinos que a corto plazo se adaptaron perfectamente a los gustos y a la técnica franceses. A partir de 1877 la presencia de comisionados galos es masiva en casi todos los términos de La Rioja Alta, dependiendo su mayor o menor actividad con la época del año.

Desde el primer momento el vino de La Rioja Alta es más apreciado por los comisionados franceses, que sólo acudirán a La Rioja Baja en las épocas en que el vino escasee en la Alta. Este comportamiento comercial obedece al hecho de que los vinos riojalteños satisfacían mejor los gustos del consumidor francés.

Simultáneamente a ester fenómeno comercial tiene lugar la aparición de las bodegas de almacenamiento, que intentan seguir las pautas de la rápida exportación vinícola; prueba evidente de su proliferación son los anuncios de vinos riojanos que llenan las revistas agrícolas de finales de siglo. Estas bodegas de almacenamiento nacen como una necesidad inseparable del incremento del cultivo del viñedo ante la ausencia inmediata de demanda.

Inicialmente, la mayor parte de las exportaciones de vino riojano a Francia lo fueron de vino común, ya que la elaboración de vino de calidad al estilo bordelés no tiene lugar hasta el último tercio del siglo XIX, en que algunos bodegueros se decidieron a elaborar vino con arreglo al estilo Médoc.

El gran incremento del viñedo no puede estudiarse aislado de las exportaciones a Francia y del asentamiento de los franceses en las villas riojanas, con el fin de adquirir cosechas enteras para su país. Los franceses ya se habían instalado en La Rioja en los años 1854 y 1855, coincidiendo con la crisis del oídio 
en Francia; en octubre del año 1854 los comerciantes franceses llegaron a La Rioja dispuestos a comprar todo el vino comercializable en esos momentos, incrementándose el precio del vino de tal forma que en pocas semanas llegó a triplicarse ante la abundancia de demanda, pasando en este mes de seis a veinte reales la cántara. Téngase en cuenta, por ejemplo, que en el año 1825, las exportaciones españolas de vino apenas alcanzaban el medio millón de hectólitros, mientras que en el año 1883 se superaban los catorce millones, de los cuales casi siete iban destinados al mercado francés, que en el año 1891 llegó a absorber diez millones de hectólitros de vino español.

El conjunto de las exportaciones se fue incrementando con la aparición de la filoxera en los campos franceses en el año 1865. A partir de la década de los ochenta, las exportaciones a Francia disminuyen por la lenta recuperación de sus viñedos y por la progresiva invasión filoxérica de regiones españolas tradicionalmente exportadoras.

\section{La llegada de la filoxera}

Procedentes de América, llegó a Europa en la segunda mitad del siglo XIX, arrasando los viñedos franceses en el año 1867. En 1868 apareció en Portugal, en 1875 en Alemania, en 1879 en Italia y en el mismo año en Suiza. En España los primeros focos aparecieron en Málaga en 1878 y Gerona en 1879; poco después llegaría a Galicia, detectándose su presencia en Orense en 1882.

La invasión filoxérica tomó caracteres catastróficos, ya que ninguno de los remedios ideados para contenerla dió resultado. La única solución posible era la de replantar las viñas con cepas americanas, injertando sobre ellas las antiguas variedades europeas, que producían un vino de más calidad y más apetecible para el consumo europeo.

Las nuevas plantaciones exigían capitales crecidos, lo cual sólo sería posible si se obtenían rendimientos elevados; por otra parte los vinateros franceses, replantada ya la viña que la filoxera había destruido años antes en su país, habían cesado en sus masivas importaciones que hacían posible la fácil venta de cualquier tipo de vino español. Por ello, en algunas regiones se duda mucho el volver a poner en explotación las antiguas viñas; por el momento, las viñas que se habían desarrollado al amparo de las coyunturales exportaciones a Francia, no volverán a ser puestas en explotación.

La crisis de la filoxera va a repercutir social y económicamente en el conjunto de la agricultura española, ya que en los últimos años del siglo XIX la mayor parte del agro tradicional había encontrado su salvación económica en la plantación del viñedo. La crisis hay que verla en su doble faceta, arrasamiento del viñedo y pérdida del principal cliente de nuestros vinos, Francia. Las repercusiones sociales fueron inmediatas; protestas, negativa a pagar impuestos, emigración de los centros exclusivamente vitícolas, etc. 


\section{LOS VIN̄EDOS RIOJANOS}

En el mapa correspondiente puede apreciarse cómo la filoxera afecta en primer lugar, antes de 1880, a parte de la provincia de Gerona y Málaga. En noviembre de 1888 a Albacete, Barcelona, Gerona, León, Murcia, Orense, Salamanca, Tarragona y Zamora. En abril de 1890 a Córdoba y en agosto del mismo año a Lugo. En 1891, a Baleares, Jaén y Sevilla. En 1894, a Cádiz, Lérida, Murcia y Oviedo. En 1896 a Navarra. En 1897 a Badajoz, Cáceres, Palencia y Valladolid. El resto de las provincias se verían afectadas en los años sucesivos.

En La Rioja, la filoxera es detectada por la Estación Enológica de Haro ${ }^{9}$ en junio de 1899 en el término de Sajazarra. Paulatinamente afecta a los términos vitícolas de la comarca de Haro, el mismo Haro, Anguciana, Cuzcurrita y otras localidades. En 1901 y 1902, todos los municipios de La Rioja Alta aparecen afectados, sobre todo en el verano de 1902 durante el que las temperaturas alcanzaron valores máximos.

La Rioja Baja comienza a verse afectada en 1904. En 1905, de forma sincrónica se extiende por Alcanadre, Aldeanueva y Tudelilla. En 1907 toda La Rioja ha sido invadida por la filoxera, siendo el tempranillo, garnacha y mazuelo, las cepas más afectadas, si bien fueron estas mismas variedades las que se injertaron en los sarmientos americanos.

Tras el arrasamiento que trajo consigo la filoxera, la recuperación del viñedo fue lenta, costosa y difícil. El retraso en la replantación de los viñedos habrá que buscarlo en causas económicas, demográficas e incluso sociales. El mercado francés - Francia se ha recuperado de la invasión filoxérica e incluso ha plantado nuevos viñedos en Argel-se da por perdido; hasta la crisis de la filoxera en La Rioja, de una forma u otra, el vino acabaría siendo adquirido por los representantes franceses afincados en nuestras tierras. En los años de la invasión filoxérica existió una considerable emigración en las áreas vitícolas, por lo que en el momento de la repoblación escaseó la mano de obra especializada. Finalmente, toda nueva plantación llevaría consigo una gran inversión y no generaría rendimiento hasta pasados varios años, y esto no estaba al alcance del agricultor medio, al que el arrasamiento del viñedo le había reducido al límite su exigua capitalización. Según la Estadística Vinícola de 1909 la filoxera había destruido 36.692 Has. e invadido 15.900.

La reconstrucción no fue uniforme en todas las comarcas de la antigua provincia de Logroño, ni las diferentes fuentes de información se ponen de acuerdo en la superficie reconstruida en cada uno de los años.

En 1915, Iriarte ${ }^{10}$ da diferentes cifras de extensión del viñedo en La Rio-

9. Fue creada en octubre de 1881. Se plantaron diferentes vides en su finca experimental tratando de detener el avance de la filoxera; organizó conferencias y orientó a los agricultores, etc.

10. Iriarte. Valor económico de La Rioja. 1915. Biblioteca del Instituto de Estudios Riojanos. 
ja, para antes y después de la invasión; según esta fuente en 1900 existirían 48.000 Has. y en 1909, 25.000 Has.; esta última fecha parece significar el comienzo de la reconstrucción. En 1915 se habría llegado a 30.000 Has., con una producción total de $229.000 \mathrm{Hl}$.; este bajo rendimiento se debió a que la mayor parte de los viñedos eran plantones y no se encontraban en plena producción. 


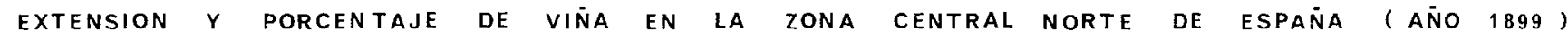

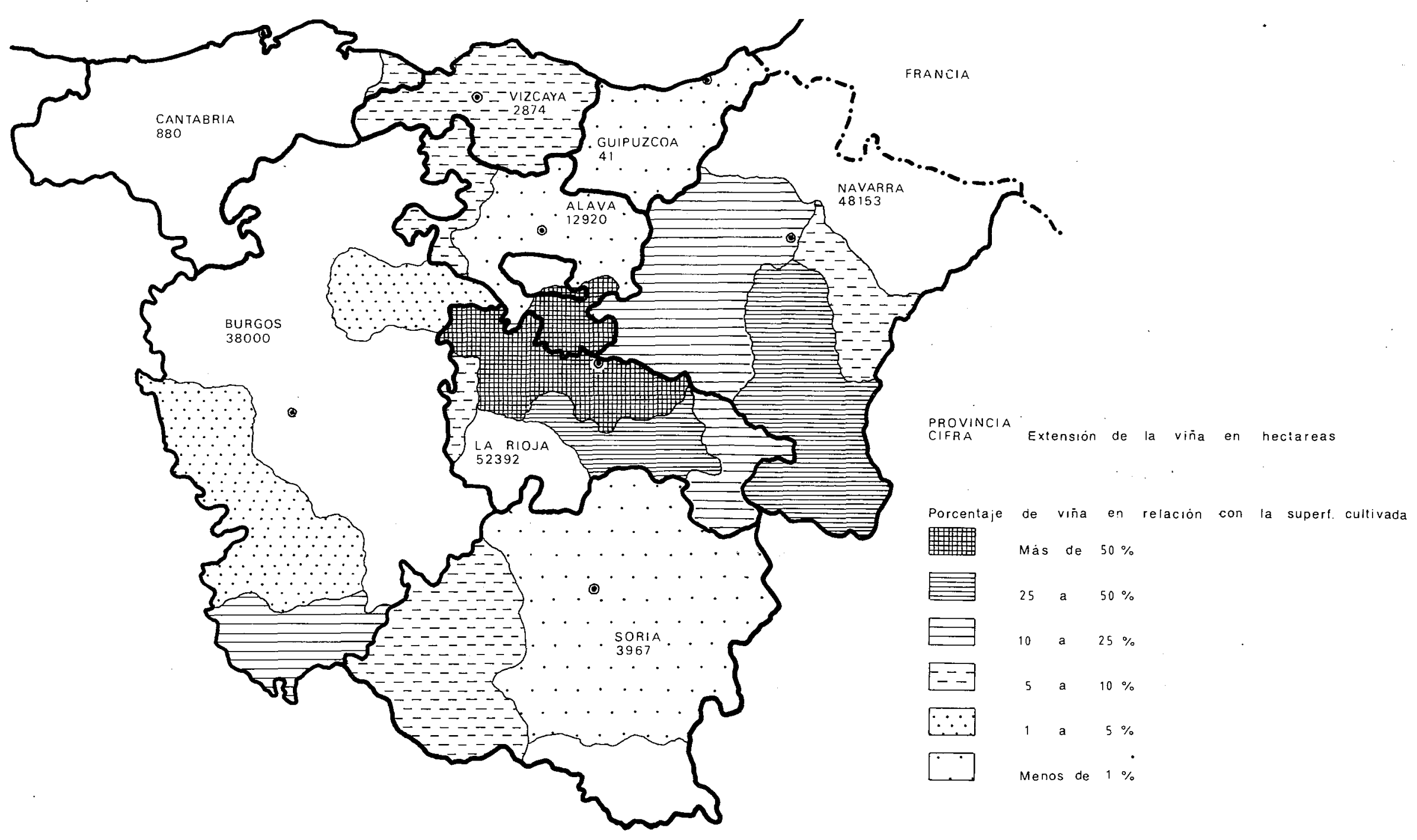


EXPORTACION DE VINO (SEGUNDA MITAD DEL SIGLO XIX)
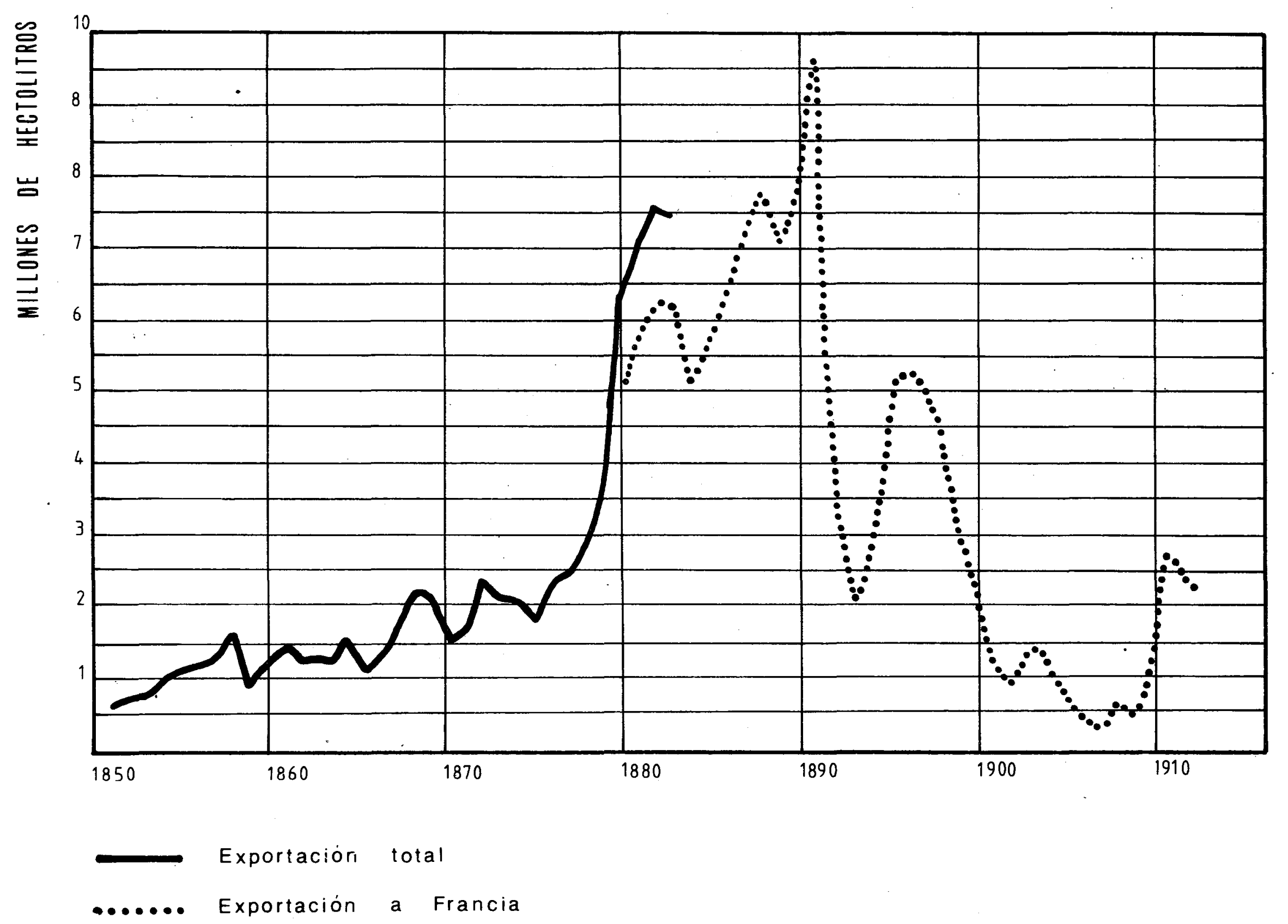
LOS VIÑEDOS RIOJANOS

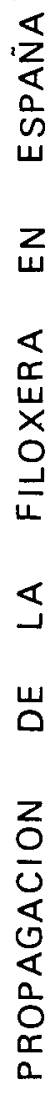

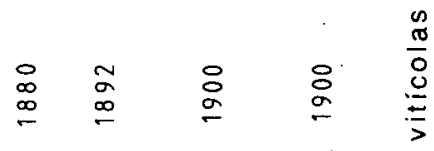

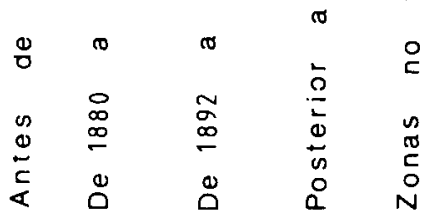
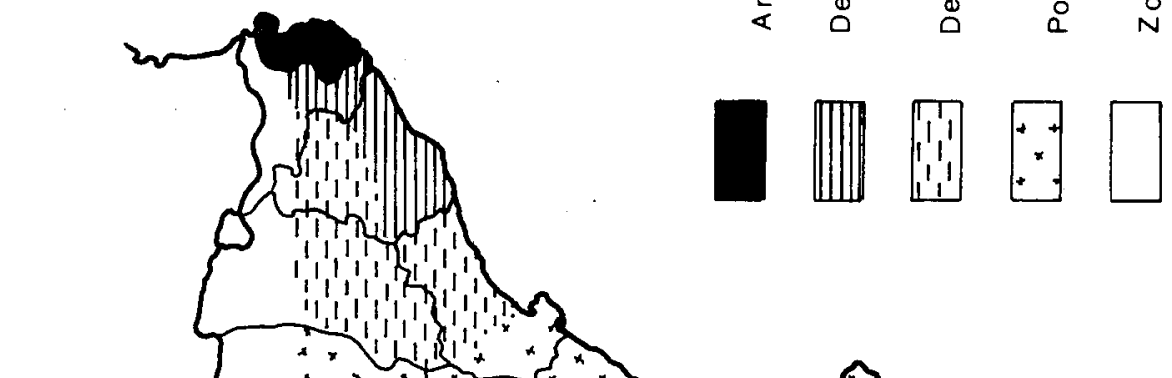
